F. Fedde: Species novas in Gardener's Chronicle, 3. ser., XLIII (1908). 365

\title{
Cll. Species novas in Gardener's Chronicle, 3. ser., XLIII (1908) descriptas
}

\author{
compilavit F. Fedde.
}

71. Mahonia arguta Hutchinson, 1. c., p. 82.

Folia 5-juga, quam inflorescentia breviora, petiolis sulcatis $17-20 \mathrm{~cm}$ longis; foliola lanceolata vel oblongo-lanceolata, subsessilia vel brevissime petiolulata, coriacea, $5-9 \mathrm{~cm}$ longa, $1-2 \mathrm{~cm}$ lata, spinoso-terminata, integra vel 1-3 spinoso-dentata, utrinque nitida, venis et venulis utrinque conspicuis. Paniculae ad apicem ramorum congestae, suberectae, laxae, $30-40 \mathrm{~cm}$ longae, ramulis elongatis $3-4$-floris compressis rigidis usque ad $4 \mathrm{~cm}$ longis. Bracteae ramorum et pedicellorum ovato-lanceolatae, 2-4 mm longae, acutae. Sepala 3 externa parva, elliptica, $3 \mathrm{~mm}$ longa, 3-nervia; 3 intermedia et 3 interna oblongo-elliptica, $6 \mathrm{~mm}$ longa, $3 \mathrm{~mm}$ lata. Petala 6, oblonga, apice bilobata, $4 \mathrm{~mm}$ longa, $3 \mathrm{~mm}$ lata. Stamina 6, filamentis $2 \mathrm{~mm}$, antheris $1-5 \mathrm{~mm}$ 'longis, Ovarium oblongum, $2 \mathrm{~mm}$ longum. Bacca globosa, $6 \mathrm{~mm}$ diametro.

Affinis $M$. paniculatae Oerst. a qua foliolis angustioribus integris vel dentibus spinosis $1-3$, minutis, reticulatione utrinque prominente, petalis minoribus recedit.

It now proves to be an undescribed species, closely allied to Mahonia paniculata 0erst. The origin of the plant is unknown, but its nearest allies are natives of Central America, where they occur at altitudes of from 8000 to 10000 feet on the slopes of volcanic mountains in Guatemala and Costa Rica.

72. Pinus Mastersiana Hayata, l. c., p. 194.

Rami teretes fusci, novelli glabri, cicatricibus perularum et foliorum notati. Folia in fasciculo quinque, aceroso-filiformia, acuta, dorso plana, in sectione triangularia, margine et in carina remote sermulata, $10 \mathrm{~cm}$ longa. Strobili erecti, ovato-cylindrici, obtusi, $14 \mathrm{~cm}$ longi. $7 \mathrm{~cm}$ lati. Bracteae minutissimae. Squamae numerosae, orbiculares v. subrhombeae, acutae, sursum reflexae, basi breviter cuneatae, lignescentes, longitudinaliter rugosae, fuscae, dispermae. Semina obovata $12 \mathrm{~mm}$ longa, $9 \mathrm{~mm}$ lata, apice leviter apiculata, exalata, compressiuscula. Testa ossea, crassa, fusca, glabra; albumen crassum, oleosum. Embryo cotyledonibus 5, verticillatis.

Formosa: In Monte Morrison, leg. G. Nakahara, 1905.

73. Pinus morrisonicola Hayata, l. c., p. 194.

Rami teretes, perularum rudimentis notati, novelli pubescentes. Gemmae ovatae, perulatae, perulis acutis, membranaceis, margine fractis. Folia in fasciculo quinque, fasciculis approximatis, acerosa, rigidula, 6 $8 \mathrm{~cm}$ longa arcuata sed non torta, apice acuta. dorso plana, facie acute carinata, in sectione triangularia, margine et carina remote serrulata. Strobili erecti, ovato-elliptici, obtusi, squamis circ. 40 compositi $7 \mathrm{~cm}$ longi, $3 \mathrm{~cm}$ lati squamis ellipticis, basi cuneatis, sursum rotundatis, le- 
viter reflexis, $3 \mathrm{~cm}$ longis, $1-5 \mathrm{~cm}$ latis, coriaceo-crassis, sublignescentibus, concavis, badio-fuscis, dispermis, sed interdum abortu monospermis. Bracteae brevissimae. Semen ovatum apice obtusum, $10 \mathrm{~mm}$ longum, $6 \mathrm{~mm}$ latum, testa coriacea pallide ferruginea glabra, ala membranacea, tenui, cultriformi, $2 \mathrm{~cm}$ longa, $8 \mathrm{~mm}$ lata.

Formosa: Shohakulin, leg. C. Owatari, 1898.

74. Tsuga formosana Hayata, 1. c., p. 194.

Ramuli novelli tenues glabri, pallide fuscentes. Gemmae perulatae, perulis obtusis integris. Folia approximata, distincte petiolata, petiolis brevibus semiteretibus, parum incurvis, linearia, 16-8 $\mathrm{mm}$ longa, 2$1,5 \mathrm{~mm}$ lata apice obtusa vel emarginata, integerrima, glabra, coriacea. Stobili ovati, $20-23 \mathrm{~cm}$ longi, $13 \mathrm{~mm}$ lati, squamis circ. 20 imbricatis, coriaceis, basi truncatis, sursum suborbicularibus, integris, $15 \mathrm{~mm}$ longis, $10 \mathrm{~mm}$ latis, substriatis, pallide fuscescentibus, bracteis brevissimis, rhomboideis, apice brevissime 2-lobatis, irregulariter dentatis. Semina parva, obovata, $4 \mathrm{~mm}$ longa, vel longiora, ala membranacea tenui cultriformi, pallide ferruginea, $7 \mathrm{~mm}$ longa.

Formosa: In Monte Morrison, leg. S. Nagasawa, 1905.

75. Juniperus morrisonicola Hayata, 1. c., p. 194.

Rami teretes; ramuli novelli virides trigoni. Folia omnia lanceolata, apice acerosa, patentiuscula, $3-4 \mathrm{~mm}$ longa, $1 \mathrm{~mm}$ lata, supra concava, glaucescentia, subtus parum carinata. Flores masculi terminales in ramulis brevissimis, ovoidei, $4 \mathrm{~mm}$ longi, $2 \mathrm{~mm}$ lati, staminibus 8 , squamis peltatis, suborbicularibus, $1,5 \mathrm{~mm}$ in diametro, antherae loculis 3 . Flores foeminei in ramulis brevissimis terminales, basi foliis squamiformibus bracteisque 6-9 oppositis suffulti, squamis intimis 3 , ovatis acutis verticillatis patentibus. Ovulum solitarium terminale a squamis intimis 3 circumdatum, oblongum, apice attenuatum. Galbulus globosus $6 \mathrm{~mm}$ diam. vel pauces longior, in apice vestigiis squamarum intimarum suffultus, siccus nigricans glaber.

Formosa: In Monte Morrisan, leg. S. Nagasawa, $190 \overline{0}$.

76. Cunninghamia Konishii Hayata, 1. c., p. 194.

Arbor ramis omnibus teretibus, glabris, foliorum spiraliter confertorum cicatricibus notatis, gemmis floriferis nudis, depresso-globosis, bracteis depresso ovatis, brevissime aristato-acutis. Folia ramorum veterorum spiraliter conferta, adnato-decurrentia, anguste lineari-falcata, incurvoerecta, acuta, dorso leviter carinata, ramulorum juvenum ascendentopatentia, longiora, anguste lineari-lanceolata, $15 \mathrm{~mm}$ longa, $2,5 \mathrm{~mm}$ lata, ad basin oblique torta, apice obtusiuscula, margine sub lente serrulata, rigida, coriacea, utrinque pagina glaucescentia, stomatibus multiseriatis instructa, octovum in annum virentia demum exarida sensim soluta. Strobili maturi ovato-globosi, $20 \mathrm{~mm}$ longi, $15 \mathrm{~mm}$ lati; squamae rotundatae, mucronatae, basi distincte unguiculatae, ungue brevi, lamina dilatata, cordata, late depresso-ovata, margine integra, lignescentes, sursum coriaceae, et marginem versus subundulatae, dorso apice leviter carinatae, glabrae; bracteae obsoletae, squamulae 3 in basi laminae squamae, distinctae, 
flmbriatae crenulatae. Semina 3 ad squamularum basin affixa, reversa, libera, ovato-elliptica, testa coriaceae, duriuscula, ala angusta cincta; embryo ignotus.

Formosa: In Monte Randaisan, Nanto, ad 7000 ped. alt., leg. N. Konishi, 1907.

77. Chamaecyparis obtusa Sieb. et Zucc. var. formosana Hayata, l. c., p. 194.

Strobili ut typicae, sed multo minores, $8 \mathrm{~mm}$ in diametro, seminibus minoribus, cum alis $2 \mathrm{~mm}$ in diametro.

Formos a:

78. Keteleeria formosana Hayata, l. c., p. 194.

Ramuli dense papilloso-puberuli. Folia laxe inserta, plana, linearilanceolata, $30 \mathrm{~mm}$ longa vel longiora, $5 \mathrm{~mm}$ lata, nervis prominentibus, margine parum deflexa, in utraque facie vernicea, subtus vix pallidiora, apice obtusa (ramuli hornotini aristato-acuta) basi in pedem brevem compressum demum contortum attenuata. Strobili erecti, cylindracei, obtusi, $9 \mathrm{~cm}$ longi, $5 \mathrm{~cm}$ lati; squamae coriaceae, ovato-rotundatae v. cordatae, superne sensim attenuatae, apice subreflexae, basi breve unguiculatae, extus longitudinaliter striatae, puberulae, margine tenues irregulariter serrulatae; bracteae squamis duplo breviores, spathulatae, membranaceae, dorso fuscae, apice cuspidatae vel trifidae, irregulariter serrulatae, ad medium contractae. Semina pallide fulva basi acuta, cum ala concolore cultriformi, squamis aequilonga. Semen cum ala $27 \mathrm{~mm}$ longum.

Formosa: Shinjuki, Shinkocho, leg. N. Konishi, 1902.

79. Malvastrum hypomadarum Sprague, 1. c., p. 394.

Frutex vix $1 \mathrm{~m}$ altus, ramis gracilibus densiuscule stellato-pubescentibus. Stipula patentes, oblique lanceolatae, $3-5 \mathrm{~mm}$ longae, ciliatae. Petioli circiter $6 \mathrm{~mm}$ longi. Folia trilobata, $2,5-4 \mathrm{~cm}$ longa et subaequilata, basi conspicue cuneata, utrinque pilis simplicibus et stellatis parce molliter pubescentia, nervis supra impressis subtus prominentibus, lobis oblongis vel in basin angustatis, medio quam lateralibus majore, dentato-serratis praesertim superne, dentibus inferioribus patulis vel leviter retro curvatis. Pedunculi 1-3-flori, ei axillarum superiorum plus minus contracti, inferiorum usque ad $1-3 \mathrm{~cm}$ longi, omnes ut pedicelli calycesque extra stellato-pubescentes. Ramuli accessorii 1 vel 2 ex iisdem axillis orti, 4-7 cm longi, foliati, 2-5-flori. Pedicelli 1,3-2 cm longi, $3 \mathrm{~mm}$ infra apices articulati. Bractea $\theta$ spathulato-lineares, 6-8 $\mathrm{mm}$ longae, $1 \mathrm{~mm}$ latae, longe ciliatae, extra puberulae, intra glabrae. Calyx intus minute appresse pubescens; tubus $3 \mathrm{~mm}$ longus, circiter 20-nervius; lobi ovati, acuminati, $6 \mathrm{~mm}$ longi, trinervii vel indistincte quinquenervii, ciliati, nervis lateralibus in tubum separatim currentibus. Corolla 2,5$4 \mathrm{~cm}$ diametro; petala oblique obovata, retusa, alba circa basin purpureomaculata, inferne dense ciliata, intus inferne stellato-pubescentia vel puberula. Columna staminea tota $1 \mathrm{~cm}$ supra invertionem petalorum producta, medio dense stellato-hirsuta. Carpella apice longe stellato- 
hirsuta, ceterum glabra; stylus $1,5 \mathrm{~mm}$ supra basin articulatus, ramis purpureis.

Planta capensis, per saeculum in hortis anglicis culta, loco natali exacto incognito.

Affinis $M$. divaricato Gray et Harv., a quo foliis serratis nec crenatis, carpellis apice tantum stellato-hirsutis ceterum calois differt.

\title{
Clll. Neue Arten aus: A. v. Hayek, Flora von Steiermark.
}

\author{
Band I, Heft 7 (1909), pp. 481-560.
}

56. Cardamine Clusiana (Rchb.) v. Hayek, l. c., p. 491.

Dentaria Clusiana Rchb., Fl. Germ. exc., 677 (1832). - Dentaria digitata Lam., Encycl, meth., II, 267 (1786); Koch, Syn., ed. 2, 48 (1843); Maly, Fl. Steierm., 195 (1868). - Cardamine digitata 0. E. Schulz in Englers bot. Jahrb., XXXII, 373 (1903), nicht Richardson in Franklin, Journ. App., VII, 743 (26) [1823]. - Dentaria pentaphyllos $\beta$. u. $\gamma$. L., Sp. pl., ed. 1, 654 (1753). - Dentaria pentaphyllos Scop., Fl. Carn., ed. 2, II, 20 (1772).

57. $\times$ Draba ficta Camus in Journ. de Bot., 1898, p. 169 (nom. nud.); v. Wettstein apud v. Hayek, l. c., p. 512 (descr.).

Draba aizoides $\times$ Sauteri Wettst. in Herbar Halacsy und in Hayek, Fl. Steierm., I, 512 (1909). - Perennis, dense vel laxe caespitosa, caudiculos elongatos rosulas ferentes emittens. Caulis erectus, $2-3 \mathrm{~cm}$ altus, glaber. Folia rosulam densam componentia, obverse lanceolata, acutiuscula, basin versus sensim attenuata, setoso-ciliata. Petala late obovata, 5-6 $\mathrm{mm}$ longa, flava. Stamina petalis multo breviora. Siliculae late ellipticae, 4-5 $\mathrm{mm}$ longa et $2 \mathrm{~mm}$ lata, utrinque breviter acuminatae, stylo $0,5-1 \mathrm{~mm}$ longo, pedunculis circiter aequilongis erecto-patentes. - Unterscheidet sich von Draba aizoides durch die kurzen Staubblätter, die breiteren, kürzer bespitzten Schötchen, den kurzen Griffel und die gegen die Spitze etwas verbreiterten Blätter, von Draba Sauteri, der der Bastard viel näher steht, durch die schmäleren, verlängerten Blätter, schmälere Schötchen und etwas grössere Blüten. - Steiermark: Unter den Stammeltern auf dem Hochschwab (Wettstein!).

58. Draba aizoides L. var. $\beta$. hispidula v: Hayek, l. c., p. 513.

Draba Beckeri Hayek in Öst. bot. Zeitschr., LI (1901), 300, nicht A. Kerner. - Schötchen besonders am Rande mit einzelnen steifen anliegenden Börstchen besetzt. - Steiermark: Hier und da, besonders in tieferen Lagen, meist unter der kahlfrüchtigen Form. Auf dem Lantsch (Wettstein!), der Krebenze bei St. Lambrecht (Strobl); auf dem Donatiberge bei Rohitsch (Alexander!).

59. $\times$ Draba Sturii (D. fadnitzensis $\times$ tomentosa) Strobl in v. Hayek, l. c., p. 517 . 